\title{
Estacionalidad, eficiencia termorreguladora de Aspidoscelis lineatissima (Sauria: Teiidae) y la calidad térmica del bosque tropical caducifolio en Chamela, Jalisco, México
}

\section{Seasonality, thermoregulation effectiveness of Aspidoscelis lineatissima (Sauria: Teiidae) and the thermal quality of a seasonally dry tropical forest in Chamela, Jalisco, Mexico}

\author{
Juan C. Navarro-García ${ }^{1}$, Andrés García ${ }^{2 *}$ y Fausto R. Méndez de la Cruz ${ }^{3}$ \\ ${ }^{1}$ Posgrado en Ciencias Biológicas, Instituto de Biología, Universidad Nacional Autónoma de México, Apartado postal 70-153, 04510 México, D.F., \\ México. \\ ${ }^{2}$ Estación de Biología Chamela, Instituto de Biología, Universidad Nacional Autónoma de México. Apartado postal 21. San Patricio, 48980 Melaque, \\ Jalisco, México. \\ ${ }^{3}$ Departamento de Zoología, Instituto de Biología, Universidad Nacional Autónoma de México, Apartado postal 70-153, 04510 México, D.F, México, \\ D.F, México. \\ *Correspondencia: chanoc@ibiologia.unam.mx
}

\begin{abstract}
Resumen. El estudio de la termorregulación del género Aspidoscelis desde la perspectiva del individuo y la precisión de éste para mantener su temperatura corporal dentro de los intervalos de temperatura preferida es escaso. Aquí registramos la eficiencia en la termorregulación de A. lineatissima en el bosque tropical caducifolio (BTC) en Chamela y el efecto de la estacionalidad en la calidad térmica del ambiente. La temperatura corporal $\left(T_{c}\right)$ de $A$. lineatissima varió estacionalmente (31.14 y $33.82^{\circ} \mathrm{C}$ en secas y lluvias). La temperatura seleccionada $\left(T_{\text {sel }}\right)$ en el laboratorio varió de $33.27^{\circ} \mathrm{C}$ en secas a 30.50 ${ }^{\circ} \mathrm{C}$ en lluvias. Las temperaturas operativas promedio $\left(T_{o}\right)$ fueron muy similares en cada estación $\left(29.30^{\circ} \mathrm{C}\right.$ y $\left.29.34^{\circ} \mathrm{C}\right)$, al igual que los índices de precisión en la termorregulación $\left(d_{b}\right)$, de calidad térmica del ambiente $\left(d_{e}\right)$ y la eficiencia en la termorregulación (E). Los resultados sugieren que la estacionalidad sólo tiene un efecto significativo en la $T_{\text {sel }}$. El valor de $T$ registrada en $A$. lineatissima es similar a la de otras especies del género. A pesar de la baja calidad térmica del BTC, A. lineatissima resultó ser una especie con eficiencia relativamente alta en su termorregulación, la cual puede estar relacionada con el tipo de forrajeo y su alto riesgo de depredación.
\end{abstract}

Palabras clave: Aspidoscelis lineatissima, termorregulación, estacionalidad, bosque tropical caducifolio, Chamela, México.

\begin{abstract}
Studies of thermoregulation in Aspidoscelis from the individual perspective and the accuracy of body temperatures relative to a range of preferred temperatures are scare. We report the effectiveness of temperature regulation of A. lineatissima in the seasonally dry tropical forest (SDTF) of Chamela, Jalisco, Mexico, and seasonal differences in the thermal quality of the environment. Body temperature $(T)$ of $A$. lineatissima varied seasonally $\left(31.14\right.$ and $33.82^{\circ} \mathrm{C}$ in the dry and wet seasons). The preferred temperatures $\left(T_{\text {se }}\right)^{\mathrm{C}}$ varied from $33.27^{\circ} \mathrm{C}$ in the dry season to $30.50^{\circ} \mathrm{C}$ in the wet season. The operative null temperatures $\left(T_{o}\right)$ were similar in both seasons $\left(29.30^{\circ} \mathrm{C}\right.$ and $\left.29.34^{\circ} \mathrm{C}\right)$, as suggested by the indices of thermoregulatory precision $\left(d_{b}\right)$, thermal quality of habitat $\left(d_{e}\right)$ and the effectiveness of temperature regulation (E). Results suggest that seasonality affects only $T_{s e l}$. The recorded $T_{c}$ is similar to that reported in other species in the genus. Despite the low thermal quality of SDTF, the effectiveness of temperature regulation of $A$. lineatissima is relatively high and could be related to its high predation risk, territoriality, and foraging mode.
\end{abstract}

Key words: Aspidocelis lineatissima, thermoregulation, seasonally tropical dry forest, Chamela, México.

\section{Introducción}

El calor es una de las fuentes de energía más importantes en todos los procesos biológicos, por lo que los ectotermos,

Recibido: 22 mayo 2007; aceptado: 23 enero 2008 como los reptiles, han desarrollado varias estrategias para la obtención de este recurso a través de la termorregulación (Huey, 1982). Así, el estudio de la termorregulación en reptiles es esencial para entender la ecología y distribución de las especies (Huey, 1982; Shine y Kearney, 2001; Shawn et al., 2002; Seebacher y Shine, 2004). Debido a su importancia, se ha desarrollado un protocolo (Hertz et 
al., 1993) para evaluar la eficiencia de la termorregulación (E) en ectodermos con base en la calidad térmica del hábitat desde la perspectiva del individuo y en la precisión de éste para mantener su temperatura corporal dentro de los intervalos de temperatura preferida. Un valor de $\mathrm{E}$ igual o cercano a 0 indicará que los organismos no están termorregulando eficientemente por lo que se comportan como termoconformistas, mientras que un valor cercano a 1 indicará un alta eficiencia termorreguladora del organismo, considerándose una eficiencia intermedia cuando el valor de E sea 0.5. Este método ha facilitado la caracterización cualitativa y cuantitativa de la termorregulación en diversas especies de lagartijas en temas como la selección del hábitat (Bauwens et al., 1996; Christian y Weavers, 1996; Grbac y Bauwens, 2001; Shawn et al., 2002), repartición de recursos (Hertz, 2004), termorregulación nocturna (Kearney y Predavec, 2000) y el efecto de la estacionalidad en los mecanismos conductuales de termorregulación (Bauwens et al., 1996; Díaz y CabezasDíaz, 2004). Si bien el uso general de este método se ha cuestionado recientemente (Seebacher y Shine, 2004), el protocolo se considera adecuado para especies de lagartijas, en su mayoría pequeñas. La crítica se relaciona con la determinación de las temperaturas operativas nulas de la especie ectotérmica en estudio, la que presupone una capacidad calorífica nula de los individuos, por lo que debe limitarse a especies o individuos pequeños.

La precisión, exactitud y eficiencia en la termorregulación de una especie en particular estará estrechamente ligada a la calidad térmica del hábitat, siendo la termorregulación especialmente importante en ambientes de baja calidad térmica (Hertz, 1993; Shine y Kearney, 2001; Shawn et al., 2002). Así, en aquellos ambientes térmicamente heterogéneos, la termorregulación será más precisa pues las especies cuentan con más alternativas para la obtención de calor (Huey y Slatkin, 1976), mientras que en ambientes con temperaturas cercanas a las óptimas que la especie requiere, la termorregulación será efectiva y requerirá de menor esfuerzo, dedicándose la energía y el tiempo a otras actividades (Shawn et al., 2002). En ambientes térmicamente estables como los tropicales, se espera que la termorregulación no sea tan importante ya que las especies tienen una continua disponibilidad de altas temperaturas a lo largo del año y fácil acceso a una amplia variedad de microclimas (Shine y Madsen, 1996).

El presente estudio combina trabajo de campo y laboratorio para medir la calidad del hábitat del bosque tropical caducifolio (BTC) y la precisión, exactitud y eficiencia en la termorregulación de Aspidoscelis lineatissima durante la estación seca y la de lluvias. Las preguntas que deseamos contestar son: $i$ ), ies $A$. lineatissima un termorregulador eficiente?; ii), ¿es el
BTC un hábitat con calidad térmica para A. lineatissima?, y iii), ¿existe un efecto de la estacionalidad ambiental en la calidad térmica del BTC y en la eficiencia de $A$. lineatissima para termorregular?

\section{Materiales y métodos}

La Estación de Biología Chamela $(\mathrm{EBCH})$ se localiza en la costa suroeste de Jalisco, México (19 $39^{\circ} \mathrm{N}, 105^{\circ}$ 03' O). El acceso a la EBCH se ubica en el Km. 59.5 de la carretera Federal 200 que corre entre Barra de Navidad y Puerto Vallarta. La temperatura y precipitación media anual es de $24.9^{\circ} \mathrm{C}$ y $750 \mathrm{~mm}$ respectivamente. La región se caracteriza por una marcada estacionalidad de las lluvias que se concentran de julio a octubre, por lo que la temporada de secas puede durar hasta 8 meses. El principal tipo de vegetación es el bosque tropical caducifolio (BTC) cuyas especies de árboles sólo presentan hojas en la temporada de lluvias. Las características generales del área pueden consultarse en múltiples fuentes (e.g. Bullock, 1986; Bullock y Solís-Magallanes, 1990; Ceballos et al., 1999, Noguera et al., 2002).

Aspidoscelis lineatissima es una lagartija mediana (LHC = 112 mm.), endémica de México; se distribuye por las tierras bajas del Pacífico desde Nayarit hasta Michoacán (García y Ceballos, 1994) y habita principalmente el BTC. Es una especie diurna, terrestre e insectívora con actividad unimodal (Casas-Andreu y Gurrola-Hidalgo, 1993; García y Ceballos, 1994) que comúnmente se observa en sitios de cobertura vegetal (Casas-Andreu y Gurrola-Hidalgo, 1993) y presenta una época de reproducción sincronizada con la época de lluvias (Ramírez-Bautista, et al., 2000).

Debido a la marcada temporalidad de las lluvias en la región de Chamela, el trabajo se realizó durante 2 visitas al área de estudio en el año 2005; una en la época seca (marzo-abril) y otra en la lluviosa (julio-agosto). Para la determinación de los requerimientos térmicos y eficiencia térmica de $A$. lineatissima, así como para estimar la calidad térmica del BTC se realizó trabajo de campo y de laboratorio durante cada visita, como se detalla a continuación y que en general siguieron las técnicas señaladas por Hertz et al. (1993).

El trabajo de campo se dividió en 2 partes, una destinada a medir la temperatura corporal $\left(T_{c}\right)$ de los individuos y del ambiente al momento de su captura (sustrato y aire, $T_{s}$ y $T_{a^{\prime}}$ respectivamente), y la otra para determinar las temperaturas operativas nulas. Se capturaron 15 individuos jóvenes a lo largo de los diferentes senderos de la $\mathrm{EBCH}$ que cruzan el BTC. Al localizar y lograr atrapar un organismo se procedió a tomar su temperatura corporal mediante un termómetro cloacal de lectura rápida Miller-Weber® con 
precisión de $0.1^{\circ} \mathrm{C}$. Además, se anotó la hora de captura, la longitud hocico-cloaca (LHC) y el peso de la lagartija, utilizando para ello una Pesola ${ }^{\circledR}$ con una capacidad de $60 \mathrm{~g}$ x 0.5g (Mathies y Andrews, 1995, 1997; Andrews et al., 1999). Para obtener las temperaturas operativas nulas $\left(T_{o}\right)$ se utilizaron 20 modelos de cobre de forma cilíndrica cuyas dimensiones fueron de $100 \mathrm{~mm}$ de largo con una base de $176.7 \mathrm{~mm}^{2}$ semejando las dimensiones del cuerpo ( $\mathrm{sin}$ cola) de un individuo adulto. Dichos modelos se colocaron de manera aleatoria en el BTC en sitios donde las lagartijas fueron observadas (hojarasca, debajo de arbustos y en la orilla de una de las veredas del BTC. Posteriormente y durante 1 día, se tomó la temperatura de cada uno de estos modelos, cada hora, desde las 10:00 hasta las 18:00 horas.

El trabajo de laboratorio incluyó 15 organismos capturados en el campo durante cada visita al área de estudio. Conforme se iba capturando a los individuos, y hasta completar la muestra necesaria de organismos (15), éstos se mantuvieron separados en cajas de plástico donde fueron alimentados con insectos de la región. Para determinar las temperaturas seleccionadas por los individuos $\left(T_{\text {sel }}\right)$ se creó un gradiente térmico para que cada individuo seleccionara la temperatura más adecuada dentro del intervalo ofrecido. Para crear el gradiente térmico se siguió la metodología sugerida por Hertz et al. (1993) en la que utiliza una caja de madera con dimensiones de 150 × 15 × $50 \mathrm{~cm}$, la cual está dividida en 5 secciones longitudinales o carriles de 15 $\mathrm{cm}$. cada uno, para evitar que los organismos se mezclaran y tener evidencia del movimiento individual de cada uno dentro del gradiente; asimismo, se colocó una malla de plástico por encima de la caja de madera para evitar que los organismos treparan y/o escaparan. Se colocaron 5 focos de 150 watts, 3 a $50 \mathrm{~cm}$ por encima y en un extremo de la caja, y 2 a la mitad, también por encima, a una altura de $100 \mathrm{~cm}$; no se colocó ningún foco al otro extremo de la caja con la finalidad de que la temperatura en este punto se igualara a la temperatura ambiental dentro del laboratorio $\left(18\right.$ a $20^{\circ} \mathrm{C}$ ) y con ello tener un gradiente 20 a $40^{\circ} \mathrm{C}$. Con la finalidad de contar con lecturas adecuadas, se permitió que las lagartijas se aclimataran al gradiente térmico durante las 2 horas previas a la toma de datos (Mathies y Andrews, 1995); la $T_{\text {sel }}$ de los organismos se registró cada hora con un termómetro de lectura rápida Miller-Weber® con precisión de $0.1^{\circ} \mathrm{C}$ durante su horario de actividad (10:00 hasta las 18:00).

Una vez obtenidos los valores de $T_{c}, T_{s}, T_{s e l}$ y $T_{o}$ se procedió a determinar los índices de precisión en la termorregulación $d_{b}$ y $d_{e}$. La $d_{b}$ es una medida de la desviación individual con respecto a $T_{c}$ y es la diferencia en el número absoluto entre la $T_{c}$ y $T_{s e l}$; el valor promedio de $d_{b}$ es un reflejo de que tanto los ectotermos experimentan una $T_{c}$ fuera del intervalo de temperaturas preferidas, donde valores altos de $d_{b}$ implican una falta en la regulación de la temperatura, mientras que valores bajos (cercanos a 0 ) implican alta precisión por parte del organismo. El índice $d_{e}$ (calidad térmica del hábitat) es la desviación en número absoluto entre $T_{o}$ y $T_{s e l}$ que es un indicador cuantitativo del promedio térmico de un hábitat desde la perspectiva de los organismos, donde valores altos indican baja calidad térmica y mientras que hábitat con un valor $d_{e}$ igual o cercano a cero son térmicamente ideales. Posteriormente, con los resultados promediados de $d_{b}$ y $d_{e}$ se calculó la eficiencia en la termorregulación (E) mediante la siguiente ecuación: $\mathrm{E}=1-\left(d_{b} / d_{e}\right)$, en donde, una $\mathrm{E}$ igual o cercana a 0 , se refiere a organismos que no están termorregulando eficientemente por lo que se comportan como un termoconformista; un valor de E cercano a 1 indica un alta eficiencia termorreguladora del organismo.

\section{Resultados}

El valor promedio de $T_{c}$ durante la estación seca fue de $31.14 \pm 3.04{ }^{\circ} \mathrm{C}\left(\mathrm{N}=15\right.$, intervalo 28.2 a $\left.33.6{ }^{\circ} \mathrm{C}\right)$, mientras que en lluvias fue de $33.82 \pm 3.87^{\circ} \mathrm{C}(\mathrm{N}=15$, intervalo 31.07 a $35.46^{\circ} \mathrm{C}$ ), sin diferencias significativas (Wilcoxon, $\mathrm{X}^{2}=$ 1.76 , gl. $1, \mathrm{P}=0.07)$. Se encontró una correlación positiva y significativa de $T_{\mathrm{b}}$ con $T_{\mathrm{s}}$ y $T_{\mathrm{a}}$ en ambas estaciones $\left(T_{s}\right.$, $\mathrm{r}^{2}=0.071, \mathrm{~N}=15, \mathrm{P}<0.0001, T \mathrm{r}^{2}=0.86, \mathrm{~N}=15, \mathrm{P}<0.0001$ en secas y $T, \mathrm{r}^{2}=0.49, \mathrm{~N}=14, \mathrm{P}<0.05 ; T_{\mathrm{a}}, \mathrm{r}^{2}=0.43, \mathrm{~N}=14$, $\mathrm{P}<0.05$ en lluvias respectivamente). La $\mathrm{T}_{\text {sel }}$ o temperatura seleccionada durante la época de secas fue de $33.27 \pm$ $3.28^{\circ} \mathrm{C}$ (intervalo 29.77 a $35.1^{\circ} \mathrm{C}$ ), un poco mayor que la de lluvias, que en promedio fue de $30.5 \pm 3.39^{\circ} \mathrm{C}(\mathrm{N}=15$, intervalo 27.3 a $33.6{ }^{\circ} \mathrm{C}$ ), diferencias que resultaron estadísticamente significativas entre estaciones (Wilcoxon, $\mathrm{X}^{2}=3.67, \mathrm{gl}$. $1, \mathrm{P}=0.00024$ ). Las temperaturas operativas nulas promedio en cada estación fueron muy similares, en la seca fue de $29.30 \pm 4.30^{\circ} \mathrm{C}$ (intervalo 21.45 a 32.7 ), mientras que en la de lluvias fue de $29.34 \pm 2.62$ (intervalo 23.15 a 32.25), no encontrándose diferencias estadísticas significativas (Wilcoxon, $\mathrm{X}^{2}=0.06$, gl. $1, \mathrm{P}>0.05$ ).

El índice de precisión en la termorregulación $\left(d_{b}\right)$ de $A$. lineatissima fue muy similar en ambas estaciones. Durante la estación seca se obtuvo un valor de $d_{b}$ de $1.36 \pm 0.39$, y de $2.02 \pm 0.63$ en la de lluvias, sin encontrarse diferencias estadísticas significativas (Wilcoxon, $\mathrm{X}^{2}=0.784, g l .1, \mathrm{P}$ $=0.43)$. La calidad térmica $\left(d_{e}\right)$ promedio del BTC durante la época seca fue de $4.02 \pm 0.49$ y de $4.48 \pm 1.2$ durante las lluvias, sin diferencias significativas (Wilcoxon, $\mathrm{X}^{2}=$ 0.62 , gl. $1, \mathrm{P}=0.53$ ). En cuanto a la eficiencia en la termorregulación (E) de A. lineatissima, se observó que las temperaturas corporales de esta especie en la estación seca se encuentran ligeramente por encima de la temperatura 
seleccionada por los organismos en laboratorio (el $40 \%$ de las $T_{c}$ se encuentran dentro de la $\left.T_{\text {sel }}\right)$, mientras que en lluvias la eficiencia termorreguladora de esta especie es mayor (el $53.33 \%$ de las $T_{c}$ se encuentran dentro del intervalo de la $\left.T_{s e l}\right)$, a su vez, las temperaturas operativas en el ambiente $T_{o}$ se distribuyeron de forma similar, presentando valores menores a $T_{c}$ y $T_{\text {sel }}$ (Fig. 1), por lo que se encontraron valores moderados de eficiencia en la termorregulación en ambas estaciones ( $E=0.66$ y 0.51 , en secas y lluvias, respectivamente).

\section{Discusión}

Nuestros resultados indican que la estacionalidad ambiental presenta un efecto significativo en la temperatura seleccionada de $A$. lineatissima siendo ésta mayor durante la estación seca, mientras que no se registraron diferencias estacionales de las temperaturas corporales, las temperaturas operativas nulas, de la calidad térmica del BTC y de la precisión y eficiencia termorreguladora de $A$. lineatissima. Los resultados también sugieren que a pesar de que la calidad térmica del BTC y la precisión en la termorregulación son bajas, la eficiencia termoreguladora de $A$. lineatissima es moderada.

El intervalo de la temperatura corporal de $A$. lineatissima determinada en este estudio es similar al registrado anteriormente para esta especie en el área de estudio por Casas-Andreu y Gurrola-Hidalgo (1993) quienes registraron una temperatura corporal promedio de $36.5^{\circ} \mathrm{C}$ (intervalo de $29-46^{\circ} \mathrm{C}$ ) para A. lineatissima. Sin embargo, ese promedio de temperatura es un poco más alto que el registrado en este estudio para ambas estaciones. Los intervalos son similares y las diferencias en los promedios registrados probablemente se deben a diferencias en la metodología utilizada en los estudios, tanto como en el tamaño de muestra (30 individuos para ambas estaciones en este estudio y 208 individuos en el trabajo previo) y la forma en que se analizaron los datos. En este estudio los promedios se analizan para cada estación por separado, mientras que en el estudio de Casas-Andreu y GurrolaHidalgo (1993) probablemente se registró la combinación del total durante todo el estudio: por otro lado, no se específica la hora en la que se colectaron con rifle la mayoría



Figura 1. Distribución de las temperaturas de Aspidoscelis lineatissima en la estación seca y la de lluvia en Chamela, Jalisco. Arriba se muestra la distribución de la temperatura corporal $\left(\mathrm{T}_{\mathrm{c}}\right)$ de los organismos en campo; abajo, la distribución de las temperaturas operativas nulas $\left(\mathrm{T}_{\mathrm{o}}\right)$ disponibles en el BTC en ambas estaciones, la barra sombreada $\left(\mathrm{T}_{\text {sel }}\right)$ la temperatura seleccionada por los organismos en el gradiente térmico en el laboratorio. 
de los ejemplares para los cuales se midió la temperatura corporal, ambiental y del sustrato. Asimismo, en dicho trabajo no se encontró una correlación estadísticamente significativa entre la temperatura corporal, del ambiente y del sustrato, mientras que el presente estudio si registra correlaciones estadísticamente significativas de dichas temperaturas en ambas estaciones. Es probable que la ausencia de una correlación significativa registrada por Casas-Andreu y Gurrola-Hidalgo (1993) se deba a que la combinación de todos los datos que obtuvieron mensualmente haya enmascarado la correlación de dichas variables y su fluctuación estacional. El presente estudio se realizó exclusivamente en BTC mientras que el trabajo previo utilizó transectos en BTC y zonas perturbadas con pastizales y cultivos donde la cobertura vegetal es mucho menor y la insolación mayor que en la BTC por lo que las temperaturas corporales fueron más altas. Otra posible explicación para dichas diferencias es que la correlación de dichas temperaturas registradas aquí, sólo sea significativa durante las primeras horas del día, como ya se sugirió para otras especies del género en las cuales la temperatura del sustrato es importante para el inicio de la actividad y de la temperatura corporal (Pianka, 1970; Porter et al., 1973; Huey, 1982, Foa et al., 1994; Foa y Bertolucci 2001; Mesquita y Colli, 2003; Winne y Keck, 2004). La mayoría de los ejemplares utilizados en este estudio se colectaron entre las 10:00 y 12:00 horas, al inicio del ciclo de actividad y quizás los ejemplares colectados por Casas-Andreu y Gurrola-Hidalgo (1993) fueron colectados a diversas horas del día cuando dicha correlación de temperatura es más débil.

La baja calidad térmica del BTC hacia $A$. lineatissima así como la baja precisión en la termorregulación de esta especie puede deberse a que la temperatura ambiental y la del sustrato durante las tardes no son de considerable importancia para el mantenimiento del ciclo de actividad de las especies del género, cuya amplitud del ciclo de actividad parece estar más relacionado con un reloj circadiano (Winne y Keck, 2004). Esto es probablemente una razón del porqué varias especies del género y de la familia Teiidae en general están activas en horas de mayor calor alcanzando en ocasiones temperaturas corporales promedio entre los 35 a $40^{\circ} \mathrm{C}$, muy por arriba de otras especies de lagartijas (Bennet y Gorman, 1979; Sartorius et al., 1999; Sproston et al., 1999; Vitt y Zani, 1996; Hatano et al., 2001; Díaz y Rocha, 2004; Simmons et al., 2005). Se sabe además que la alta movilidad característica de este género (Magnusson et al., 1985; Anderson, 1993) puede oscurecer las relaciones entre la temperatura corporal y la ambiental (Schall, 1977; Teixeira-Filho et al., 1995), lo que podría resultar en calidades térmicas bajas del ambiente y en altas eficiencias termorreguladoras por parte de las especies.

El modelo de Hertz et al. (1993) fue diseñado para determinar si una especie en particular termorregula o no, por lo que una alta eficiencia en la termorregulación indica que la especie es termorreguladora activa, lo que parece ocurrir en el caso de $A$. lineatissima. Las especies del género Aspidoscelis son forrajeras muy activas con temperaturas corporales similares a pesar de habitar ambientes térmicamente disímiles como los desiertos, los trópicos y subtrópicos (Pianka, 1970, 1977; Bennet y Gorman, 1979; Sartorius et al., 1999; Sproston et al., 1999; Vitt y Zani, 1996; Hatano et al., 2001; Díaz y Rocha, 2004; Simmons et al., 2005), por lo que las temperaturas corporales están sujetas a las temperaturas operativas y a la habilidad para termorregular, mientras que las temperaturas seleccionadas se encuentran influenciadas por la inercia filogenética. Por otro lado, las temperaturas corporales seleccionadas en el laboratorio nos brindan un panorama más amplio de cuáles serían las preferencias térmicas corporales de estar disponibles en el campo. Es probable que en el BTC existan dichas temperaturas y que aun sin poder acceder a los microclimas de mayor calidad térmica, aunado a los costos ecológicos de una termorregulación efectiva, como un alto riesgo de depredación, una marcada territorialidad y el tipo de forrajeo, A. lineatissima pueda termorregular eficientemente (Huey y Slatkin, 1976; Schall, 1977; Paulissen, 1988; Mathies y Andrews, 1997).

\section{Agradecimientos}

A la Estación de Biología Chamela del Instituto de Biología, UNAM, por las facilidades otorgadas durante la elaboración de este trabajo así como a los revisores anónimos que mejoraron el manuscrito a través de sus comentarios y sugerencias. Este trabajo fue posible en parte gracias a una beca de posgrado otorgada al primer autor por CONACyT (Núm.189404) y al financiamiento obtenido del PAPIIT por medio del proyecto IN212605 asignado al segundo autor.

\section{Literatura citada}

Andrews, R. M., F. R. Méndez-De la Cruz, M. Villagrán-Santa Cruz y F. Rodríguez-Romero. 1999. Field and selected body temperaturas of the lizards Sceloporus aeneus and Sceloporus bicanthalis. Journal of Herpetology 33:93-100.

Anderson, R. A. 1993. Analysis of foraging in a lizard, Cnemidophorus tigris: salient features and environmental effects. In Biology of whiptail lizards (genus Cnemidophorus), J. W. Wright y L. J. Vitt (eds.). Herpetologists' League special publication No. 3. p. 83- 
116

Bauwens, D., P. E. Hertz y A. M. Castilla. 1996. Thermoregulation in a lacertid lizard: the relative contributions of distinct behavioural mechanism. Ecology 77:1818-1830.

Bennett, F. A. y G. Gorman. 1979. Population density and energetics of lizards on a tropical island. Oecologia 42:339-358.

Bullock, S. 1986. Climate of Chamela, Jalisco, and trends in the south coastal region of Mexico. Archives for Meteorology Geophysics and Bioclimatology Series B 36:297-316.

Bullock, S. y A. Solis-Magallanes. 1990. Phenology of canopy trees of a tropical deciduous forest in Mexico. Biotropica 22:22-35

Casas-Andreu, G. y M. A. Gurrola-Hidalgo. 1993 .Comparative ecology of two species of Cnemidophorus in coastal Jalisco, Mexico. In Biology of whiptail lizards (genus Cnemidophorus), J. W. Wright y L. J. Vitt (eds.). Herpetologists' League special publication No. 3. p. 133-149.

Ceballos, G., A. Szekely, A. García, P. Rodríguez y F. Noguera. 1999. Plan de manejo de la Reserva de la Biosfera ChamelaCuixmala. Secretaría del Medio Ambiente Recursos Naturales y de Pesca, México D.F. 63 p.

Christian, K. A. y B. Weavers. 1996. Thermoregulation of monitor lizard in Australia: an evaluation of methods on thermal biology. Ecological Monographs 66:139-157.

Díaz, J. A. y Cabezas-Díaz, S. 2004. Seasonal variation in the contribution of different behavioural mechanisms to lizard thermoregulation. Functional Ecology 18:867-875.

Díaz, J., R. E. y F. D. H. Rocha. 2004. Thermal ecology, activity patterns, and microhabitat use by two sympatric whiptail lizards (Cnemidophorus abaetensis and Cnemodophorus ocellifer) from Northeastern Brazil. Journal of Herpetology 38:586-588.

Foa, A., G. Monteforti, L. Minutini, A. Innocenti, C. Quaglieri y M. Flamini. 1994. Seasonal changes of locomotor activity patterns in ruin lizards Podarcis sicula. Behavioural Ecology and Sociobiology 34:267-274.

Foa, A. y Bertolucci, C. 2001. Temperature cycles induce a bimodal pattern in ruin lizards: masking or clock-controlled event? A seasonal problem. Journal of Biology Rhythms 16:574-584.

García, A. y G. Ceballos. 1994. Guía de los reptiles y anfibios de la costa de Jalisco. Fundación Ecológica de CuixmalaInstituto de Biología, Universidad Nacional Autónoma de México, México, D.F. 184 p.

Grbac, I. y D. Bauwens. 2001. Constraints on temperature regulation in two sympatric Podarcis lizards during Autumn. Copeia 2001:178-186.

Hatano, F. H., D. Vrcibradic, C. A. B. Galdino, M. Cunha-Barros, C. F. D. Rocha y M. Van Sluys. 2001. Thermal ecology and activity patterns of the lizard community of the Restinga of Jurubatiba, Macaé, R. J. Revista Brasileira de Biologia 61:287-294.

Hertz, P. E., B. R. Huey y R. D. Stevenson. 1993. Evaluating temperature regulation by field-active ectotherms: the fallacy of the inappropriate question. The American Naturalist 142:796-818.

Hertz, P. E. 2004. Evaluating thermal resource partitioning.
Oecologia 2004:1432-1939.

Huey, R. B. y M. Slatkin. 1976. Cost and benefits of lizard thermoregulation. Quarterly Review of Biology 51:363-384.

Huey R. B. 1982. Temperature, physiology and the ecology of reptiles. In Biology of the Reptilia, vol. 12, C. Gans y F. H. Pough (eds.). Academic Press, London. p. 25-92.

Kearney, M. y M. Predavec, 2000. Do nocturnal ectotherms thermoregulate? A study of the temperate gecko Christinus marmoratus. Ecology 81:2984-2996.

Magnusson, W. E., L. J. D. Paiva, R. M. D. Rocha, C. R. Franke, L. A. Kasper y A. P. Lima. 1985. The correlates of foraging mode in a community of Brazilian lizards. Herpetologica 41:324-332.

Mathies, T. y R. M. Andrews. 1995. Termal and reproductive biology of high and low elevation populations of the lizard Sceloporus scalaris: implications for the evolution of viviparity. Oecologia 104:101-111.

Mathies, T. y R. M. Andrews. 1997. Influence of pregnancy on the thermal biology of the lizard, Sceloporus jarrovi: why do pregnant females exhibit low body temperatures? Functional Ecology 11:498-507.

Mesquita, D. O.y G.R.Colli. 2003. The ecology of Cnemidophorus ocellifer (Squamata, Teiidae) in a Neotropical savanna. Journal of Herpetology 37:498-509.

Noguera, F., J. H. Vega-Rivera, A. N. García-Aldrete y M. Quesada-Avendaño (eds.). 2002. Historia natural de Chamela. Instituto de Biología, Universidad Nacional Autónoma de México, México, D.F. 563 p.

Paulissen, M. 1988. Ontogenetic and seasonal comparisons of daily activity patterns of the six-lined racerunner Cnemidophorus sexlineatus (Sauria:Teiidae). American Midland Naturalist 120:355-361.

Pianka E. R. 1970. Comparative autoecology of the lizard Cneidophorus tigris in different parts of its geographic range. Ecology 51:703-720.

Porter, W. P., J. W. Mitchell, W. A. Beckman y C. B. De Witt. 1973. Behavioral implications of mechanistic ecology: thermal and behavioral modeling of desert ectotherms and their microenviroment. Oecologia 13:1-54.

Ramírez-Bautista, A., C. Balderas-Valdivia y L. Vitt. 2000. Reproductive ecology of the wipthail lizard Cnemidophorus lineatissimus (Squamata: Teiidae) in a tropical dry forest. Copeia 3:712-722.

Sartorius, S. S., L.. J. Vitt y G. R. Colli. 1999. Use of naturally rainforest by the teiid lizard Ameiva ameiva. Biological Conservation 90:91-101.

Schall, J. J. 1977: Thermal ecology of five sympatric species of Cnemidophorus (Sauria: Teiidae). Herpetologica 33:261-272.

Seebacher F. y R. Shine. 2004. Evaluating thermoregulation in reptiles: the fallacy of the inappropriately applied method. Physiological Biochemical Zoology 77:688-95.

Shawn, S., J. Sartorius, P. S. do Amaral, R. D. Durtsche, C. M. Deen y W. I. Lutterschmidt. 2002. Thermoregulatory accuracy, precision, and effectiveness in two sand-dwelling lizards under mild environmental conditions. Canadian Journal of Zoology 80:1966-1976.

Shine, R. y T. Madsen. 1996. Is thermoregulation unimportant for most reptiles? An example using water phytons (Liasis 
fuscus) in tropical Australia. Physiological Zoology 69:252-269.

Shine, R. y M. Kearney. 2001. Field studies of reptile thermoregulation: how well do physical models predict operative temperatures? Functional Ecology 15:282-288.

Simmons, P. M., B. T. Greene, K. E. Williamson, R. Powell y J. S. Parmerlee, jr. 2005. Ecological interactions within a lizard community on Grenada. Herpetologica 61:124-134.

Sproston, A., R. Glor, L.. Hartley, E. Censky, R. Powell y J. Parmerlee. 1999. Niche differences among three sympatric species of Ameiva (Reptilia:Teiidae) on Hispaniola. Journal of Herpetology 33:31-136.

Teixeira-Filho, P. F., C. F. D. Rocha y S. C. Ribas. 1995. Aspects of thermal ecology and habitat use by Cnemidophorus ocellifer (Sauria: Teiidae) in the Restinga of Barra de Marica', Rio de Janeiro. In Oecologia Brasiliensis, vol. 1. Structure, Function and Management of Brazilian Ecosystems, F. D. A. Esteves (ed.). Symposium on Ecosystems Structure, Function and Management, Rio de Janeiro, Brazil, May 2729, 1992, Institute of Biology, Federal Universidade of Rio de Janeiro. p. 155-165.

Vitt, L. J. y P. A. Zani. 1996. Ecology of the lizard Ameiva festiva (Teiidae) in Southeastern Nicaragua. Journal of Herpetology 30:110-117.

Winne, C. T. y M. B. Keck. 2004. Daily activity patterns of Whiptail Lizards (Squamata: Teiidae:Aspidoscelis): a proximate response to environmental conditions or an endogenous rhythm? Functional Ecology 18:314-321. 